\title{
EFFECT OF NON-SURGICAL PERIODONTAL THERAPY ON SALIVARY AND SERUM LEPTIN LEVELS IN PATIENTS WITH GENERALIZED STAGE II GRADE B PERIODONTITIS
}

\author{
Girish Suragimath, Divyanee Doshi, Siddharth Varma, Sameer A. Zope, Ashwinirani SR \\ Department of Periodontology, School of Dental Sciences, Krishna Institute of Medical Sciences Deemed to be University, Karad, Maharashtra, India
}

\begin{abstract}
INTRODUCTION: Leptin is a peptide-based hormone, known to maintain fat reserves, and forms an integral part of host defense. The level of leptin is found to be altered in various inflammatory disorders, including periodontitis. The outcome of non-surgical periodontal therapy (NSPT) on leptin concentrations in the saliva and serum of patients with periodontitis is yet to be determined.

ОвJеCTIVES: The aim of this study was to evaluate the effect of NSPT on salivary and serum leptin levels in patients suffering from generalized stage II grade B periodontitis.

MATERIAL AND METHODS: This randomized, controlled, clinical trial was conducted on forty-five subjects with normal body mass index, suffering from periodontal disease. Periodontal parameters (gingival index, probing pocket depth, plaque index, and clinical attachment loss) were recorded in a structured proforma. Salivary and serum leptin levels at baseline and three months post-NSPT (after scaling and root planning, followed up with oral hygiene instructions to patients) were analyzed using enzyme-linked immunosorbent assay. Descriptive statistics and paired $t$-test were applied to compare the variables. $P$-value $<0.05$ was considered statistically significant.

RESULTS: The mean age of participants was $40.6 \pm 4.2$ years, with a male to female ratio $1: 1.2$. Significant improvements in the periodontal parameters were observed three months post-therapy $(p<0.001)$. The difference in serum leptin levels before and after NSPT was statistically significant with $p<0.001$, whereas the difference in salivary leptin (SAL) levels before and after NSPT was negatively significant with $p<0.001$.

ConcLusions: The serum leptin level significantly reduced, while SAL level significantly increased after NSPT. In other words, SAL levels of leptin can be used as a biomarker for periodontal health and disease.
\end{abstract}

KEY WORDs: leptin, non-surgical periodontal therapy, periodontitis, saliva, serum.

J Stoma 2021; 74, 2: 84-88

DOI: https://doi.org/10.5114/jos.2021.106267

\section{INTRODUCTION}

Periodontal disease (PD) is an inflammatory and immune modulatory condition that affects tissues supporting the teeth. The complex interplay between the host response against various microorganisms and their by-products leads to advancement of PD. There has been renewed interest in how periodontitis can affect cellular and molecular components of blood $[1,2]$. The periodontal treatment may alter the levels of these molecular components improving periodontal health. One such component is leptin, with its levels known to vary depending on the severity of inflammatory disorder, including PD [3].
JOURNAL OF STOMATOLOGY CZASOPISMO STOMATOLOGICZNE
AdDress For CORRESPONDENCE: Dr. Girish Suragimath, Department of Periodontology, School of Dental Sciences, Krishna Institute of Medical Sciences Deemed to be University, Karad 415539, Maharashtra, India, e-mail: drgirishsuragimath@gmail.com

ReCeIVed: 27.01.2021 • ACCEPTED: 27.03.2021 • Published: 04.06.2021 
Leptin is a pleiotropic hormone produced by adipocytes and various other tissues, including gingiva. It reduces appetite and stimulates sympathetic nervous system, lowering adipose tissue mass. Leptin has also been classified as a cytokine because it is structurally similar to the long chain helical cytokine family [3]. It stimulates the immune system and increases proinflammatory cytokine production [4]. A positive correlation in serum leptin concentration according to PD severity was reported [5]. A previous study found the presence of leptin in human saliva, which showed a negative correlation of salivary leptin (SAL) in periodontitis [6].

Non-surgical periodontal therapy (NSPT) is a quick and effective method to stop the spread of periodontal infection without the need for an invasive surgery [6]. The NSPT involves scaling and root planing (SRP) procedures, along with oral hygiene instructions for patients to follow. SRP is a regular procedure in NSPT that is effective in controlling gingivitis and preventing its progression to $\mathrm{PD}$, and is indicated by a reduction in inflammatory markers of serum and saliva. Currently, there is limited literature on the effect of NSPT on serum and SAL levels.

\section{OBJECTIVES}

The present study assesses the concentration of leptin in the saliva and serum of patients with generalized periodontitis, and evaluates the effect of NSPT on serum leptin and salivary leptin levels.

\section{MATERIAL AND METHODS}

This prospective clinical trial was conducted at the Department of Periodontology, School of Dental Sciences, Karad, Maharashtra, India, from December 2018 to August 2019. The protocol and materials used were approved by the ethical board and cleared by the Krishna Institute of Medical Sciences "Deemed to be University” (KIMSDU), Karad (Ref No. KIMSDU/IEC/02/2018, dated on March 13, 2018). Patients were informed about the purpose of the study, and a written, informed consent was obtained before enrollment. The study population, recruited by convenient sampling included 45 participants, with generalized stage II grade B periodontitis and moderate rate of progression, as per criteria of the American Academy of Periodontology (AAP) [7]. The primary AAP criteria for grade B periodontitis is having a direct evidence of radiographic bone loss or clinical attachment loss (CAL) $>2.0 \mathrm{~mm}$ over 5 years, or a direct evidence of bone loss, shown by destruction commensurate with biofilm deposits [7]. The subjects were between 35-65 years of age, having minimum 20 teeth, and a normal body mass index (BMI; 18.5 to $25 \mathrm{~kg} / \mathrm{m}^{2}$ ) according to the World Health Organization's criteria [8].
Pregnant and lactating females, subjects using tobacco in any form, those who had received periodontal treatment in the preceding 12 months, or those on any medication in the past six months were excluded. Patients with any systemic diseases (cardiovascular diseases, rheumatoid arthritis, osteoarthritis, and diabetes mellitus), or who had suffered from any recent infections were also excluded from the study.

Demographic data, socio-economic status, and lifestyle habit of the participants were recorded using standard proforma.

Primary outcome was the estimation of salivary and serum leptin levels before and after three months of NSPT. Secondary outcomes included measurement of parameters, including gingival index (GI), plaque index (PI), probing pocket depth (PPD), and CAL and comparison of leptin levels between the genders at baseline (before NSPT) and three months after NSPT.

All the patients enrolled in the study were subjected to periodontal analysis, which included gingival index [9] and plaque index [10]. Probing pocket depth (PPD) and clinical attachment level (CAL) were recorded to the nearest millimeter, with a periodontal probe at six sites (mesiobuccal, buccal, distobuccal, mesiolingual, lingual, and distolingual) on all teeth, excluding the third molars [11]. The periodontal examination was carried out by one independent calibrated examiner (DD) throughout the study. Under aseptic conditions, two milliliters of blood was drawn from the antecubital fossa by venipuncture, using a 20-guage needle and collected into a plain vacutainer. The blood samples were screened for human immunodeficiency virus, hepatitis B and hepatitis $C$ viruses. Blood samples were allowed to clot at a room temperature (1 hour), and the serum was extracted by 3,000 rpm centrifugation for five minutes. Half milliliter of the extracted serum was directly transferred to a plastic vial, assigned a tracking number, and stored at $-80^{\circ} \mathrm{C}$ till the time of assay.

Two milliliters of whole, unstimulated saliva was collected (between 10.00 a.m. to 12.00 p.m.) two hours after the last meal. According to the modified draining method, patients were asked to rinse their mouth thoroughly with distilled water before collection [12]. They were requested to drop their head down to let the saliva pool in the floor of the mouth and asked to expectorate the saliva. The desired volume ( $2 \mathrm{ml})$ of saliva was then pipetted into a test tube. Saliva samples were centrifuged at 4,000 rpm for $10 \mathrm{~min}$ to remove cell debris, and $0.5 \mathrm{ml}$ of the supernatant was transferred into a $1.5 \mathrm{ml}$ plastic vial and stored at $-80^{\circ} \mathrm{C}$.

All NSPTs of patients were performed by the same experienced periodontologist to avoid bias after collection of blood and saliva samples at baseline by a trained operator. NSPT included scaling and root planing performed in a single sitting, using an ultrasonic scaling unit (Williams's periodontal probe Hu-Friedy, Rotterdam, The Netherlands; SONIC flex air scaler, KaVo, 
Biberach, Germany) and curettes (Standard Gracey curettes, Hu-Friedy, Chicago, IL, USA). Following the procedure, patients were instructed to maintain a strict oral hygiene. The subjects were recalled after three months, and clinical examination was carried out. Blood and saliva samples were collected, and leptin levels were measured using an enzyme-linked immunosorbent assay (ELISA) kit.

The levels of serum and SAL were determined using Ray Bio ${ }^{\circledR}$ ELISA kit (3607 Parkway Lane, Suite. 200, Norcross, GA, USA), which contained biotin antibody, HRP-streptavidin solution, and TMB substrate. The assay was carried out as per product instructions, and the

TABLE 1. Socio-demographic characteristics of the study population

\begin{tabular}{|c|c|}
\hline \multicolumn{2}{|l|}{ Characteristics } \\
\hline \multicolumn{2}{|l|}{ Gender, $n(\%)$} \\
\hline Male & $24(53.3)$ \\
\hline Female & $21(46.7)$ \\
\hline \multicolumn{2}{|l|}{ Age (years), mean $\pm S D$} \\
\hline Male & $40.62 \pm 3.84$ \\
\hline Female & $40.57 \pm 4.82$ \\
\hline BMI $\left(\mathrm{kg} / \mathrm{m}^{2}\right)$, mean $\pm \mathrm{SD}$ & $20.62 \pm 1.16$ \\
\hline
\end{tabular}

TABLE 2. Comparison of serum and salivary leptin concentrations by paired t-test

Concentration Pre-NSPT (pg/ml) Post-NSPT (pg/ml) p-value

\begin{tabular}{|l|c|c|c|}
\hline Serum leptin & $1131.14 \pm 134.90$ & $345.86 \pm 35.25$ & $<0.001^{*}$ \\
\hline Salivary leptin & $29.58 \pm 9.34$ & $72.87 \pm 17.99$ & $<0.001^{*}$ \\
\hline
\end{tabular}

NSPT - non-surgical periodontal therapy. * *ignificant at $p<0.05$

TABLE 3. Comparison of periodontal parameters (mean $\pm \mathrm{SD}$ ) by paired t-test

\begin{tabular}{|c|c|c|c|}
\hline Clinical parameter & Pre-operative & Post-operative & $p$-value \\
\hline $\mathrm{PI}$ & $2.23 \pm 0.25$ & $0.80 \pm 0.44$ & $<0.001^{*}$ \\
\hline Gl & $2.28 \pm 0.26$ & $0.48 \pm 0.21$ & $<0.001^{*}$ \\
\hline PPD (mm) & $5.62 \pm 0.61$ & $2.15 \pm 0.15$ & $<0.001^{*}$ \\
\hline CAL (mm) & $4.54 \pm 0.21$ & $2.11 \pm 0.15$ & $<0.001^{*}$ \\
\hline
\end{tabular}

absorbance at $570 \mathrm{~nm}$ of the substrate color reaction was read on ELISA reader (Lisa Quant TS, Tulip Diagnostics Pvt. Ltd., Goa, India). The salivary and serum leptin values were calculated using a quadratic regression equation, and the total leptin levels were recorded in pictograms (pg).

The data obtained was recorded using Microsoft Excel 2010. All analysis were performed using statistical software IBM SPSS Statistics for Windows, version 20.0 (IBM Corp., Armonk, NY, USA), and $p$-value $<0.05$ was considered statistically significant. The descriptive statistics were expressed as mean \pm standard deviation (SD) for serum leptin, SAL as well as for PPD, CAL, PI, and GI. Pre- and post-NSPT comparison for each parameter was performed by paired $t$-test. Comparison between gender for leptin levels at reference point, and 3 months post-NSPT was carried out by unpaired $t$-test.

\section{RESULTS}

A total of 45 patients were included in the study, with a mean age of $40.6 \pm 4.2$ years and a male to female ratio of $1: 1.2$ (Table 1 ). There was significant reduction in serum leptin levels from baseline (1131.14 $\pm 134.91 \mathrm{pg} / \mathrm{ml}$ ) to three months post-NSPT (345.86 \pm $35.25 \mathrm{pg} / \mathrm{ml})(p<0.001)$. A significant elevation in SAL levels was noted from baseline $(29.58 \pm 9.34 \mathrm{pg} / \mathrm{ml})$ to three months post-NSPT $(72.87 \pm 17.99 \mathrm{pg} / \mathrm{ml})$ $(p<0.001)$ (Table 2).

Comparison of the clinical parameters at reference point and post-NSPT showed significant differences with $p$-value $<0.001$ (Table 3 ). There was not significant difference in serum leptin levels between genders at baseline and post-NSPT (Table 4).

\section{DISCUSSION}

Periodontitis is an inflammatory condition affecting the periodontium, i.e., tooth supporting structures. It has been reported that PD aggravates various systemic diseases and conditions, including diabetes mellitus and cardiovascular diseases, and the treatment of PD prevents progression of these conditions [11-13]. PD alters serum leptin levels, thereby affecting fat metabolism [13]. Therefore, the current study was planned to estimate and compare the serum and SAL levels pre- and three months postNSPT in patients with generalized stage II grade B periodontitis.

TABLE 4. Comparison of pre-NSPT and post-NSPT serum leptin $(\mathrm{pg} / \mathrm{ml})$ and change in serum leptin among genders by unpaired t-test

\begin{tabular}{|l|c|c|c|}
\hline \multicolumn{2}{|c}{ Male } & Female & $p$-value \\
\hline Pre-operative leptin levels $(\mathrm{pg} / \mathrm{ml})$ & $1140.69 \pm 119.29$ & $1119.90 \pm 154.28$ & 0.647 \\
\hline Post-operative leptin levels $(\mathrm{pg} / \mathrm{ml})$ & $353.14 \pm 36.64$ & $337.29 \pm 32.51$ & 0.176 \\
\hline
\end{tabular}


Studies have shown that confounding factors, such as smoking and BMI level, affect leptin levels in serum and gingival crevicular fluid (GCF). Bozkurt et al. reported that the leptin levels in GCF of smokers were lower compared to non-smokers, and that smoking could interrupt leptin level regulation [13]. Increase in BMI level has also shown to progressively increase serum leptin concentration [14]. Hence, this study included only patients with normal BMI. Those suffering from any systemic diseases and smokers were excluded for the same reason.

Salivary leptin, a member of IL-6 family, prevents a decrease in the synthesis of mucin in salivary glands after the activation of lipopolysaccharides. Thus, the salivary leptin plays a role in preventing the growth of bacteria altering mucin. Salivary leptin levels were altered in many inflammatory disorders, i.e., form cardiac disorders to periodontitis. The severity of disease as opposed to healthy controls was studied by means of leptin levels in serum or saliva [16-19].

There was a significant reduction in serum leptin levels $(p<0.001)$ post-NSPT compared to baseline. These findings were in accordance with studies conducted by Shimada et al. and Purwar et al. [6, 15]. Moreover, the results of the present study were also in agreement with previous studies, which reported that serum leptin levels were higher in periodontitis patients [16, 17]. Leptin levels have been reported to increase in cardiovascular diseases (CVD) patients [18-20].

The raised serum leptin level in periodontitis subjects may be due to stimulatory action of lipopolysaccharides and elevated cytokines (TNF- $\alpha$ and IL-1), resulting in an increase in leptin production with its subsequent release into the systemic circulation [21]. The elevation in serum leptin levels could also be a fundamental part of the immune response and host defense mechanism [22]. Inflammation leads to elevated vascular endothelial growth factor (VEGF), which increases the vascular network in the gingiva, resulting in leptin being withdrawn from the gingiva into circulation [23]. As shown in various studies, the raised serum leptin levels in periodontitis patients increases the risk of cardiovascular diseases due to formation of atherogenic plaques from the stimulated inflammatory signaling pathway [24,25]. Patients with cardiovascular diseases were not included in the present study; although, based on previous observations, it can be assumed that elevated serum leptin concentrations in patients with periodontitis can lead to adverse cardiovascular outcomes.

The current study used salivary samples for the estimation of leptin levels instead of GCF to overcome challenges, including contamination with blood, saliva, or plaque, and evaporation frequently associated with GCF. Saliva collection methods are non-invasive, cost-effective, fast, and not technique-sensitive compared to GCF. Previous studies have also shown that salivary analysis reveals current disease activity, making it a potentially viable alternative [6].
In the current study, the SAL level at baseline was lower compared to the value obtained after three months post-NSPT $(p<0.001)$. Previous studies have mentioned a possibly protective role for leptin in maintaining periodontal health [24]. Our results concur with other studies, demonstrating a negative correlation between SAL levels and PD $[6,26]$. In contrast, another study showed that leptin was present in both healthy and inflamed human gingival tissues, but its concentration decreased with worsening of periodontal condition [27].

A brief review of previous published literature on salivary leptins indicated that leptins acts as circulating cytokines (mediating inflammatory process) and play an important role in mucosal host defense. Periodontal inflammation-induced cytopathic changes, and results in an increased expression of leptin receptors, which can be a diagnostic marker as used in earlier studies [25-29]. The importance of lower levels of SAL in periodontitis at baseline may be attributed to the increased binding of leptin to these receptors $[28,29]$. The leptin causes induction of human peripheral mononuclear cells, chemotaxis, and stimulation of polymorphonuclear cells. Therefore, a higher local concentration of leptin also protects the host from any infections and inflammation, and reflect a fair prognosis periodontal therapy [26, 30-32].

Periodontitis is characterized by elevated PI, GI, and the presence of periodontal pockets with loss of attachment, as observed at baseline in our study. There was a significant improvement in PI, GI, PPD and CAL levels three months post-NSPT. These findings were also concurrent with other studies that showed an improvement in periodontal parameters, as periodontal inflammation reduced $[6,26,27]$.

The current study was limited in its small sample size and short follow-up of only three months. A longer follow-up period and larger sample size would provide a more reliable evidence about changes in serum and salivary leptin levels in patients with periodontitis following non-surgical periodontal therapy.

\section{CONCLUSIONS}

In the current study, NSPT improved the patients' oral health by decreasing plaque levels, gingivitis, PPD, and facilitating an improvement in CAL. Moreover, NSPT was able to reduce serum leptin and increase salivary leptin towards health. Therefore, SAL levels could serve as a biomarker to evaluate the status of periodontitis.

\section{CONFLICT OF INTEREST}

The authors declare no potential conflicts of interest with respect to the research, authorship, and/or publication of this article. 


\section{References}

1. Albert CM, Ma J, Rifai N, et al. Prospective study of C-reactive protein, homocysteine, and plasma lipid levels as predictors of sudden cardiac death. Circulation 2002; 105: 2595-2599.

2. Ridker PM, Brown NJ, Vaughan DE, Harrison DG, Mehta JL. Established and emerging plasma biomarkers in the prediction of first atherothrombotic events. Circulation 2004; 109 (25 Suppl 1): IV6-IV19.

3. Zhang Y, Proenca R, Maffei M, Barone M, Leopold L, Friedman JM. Positional cloning of the mouse obese gene and its human homologue. Nature 1994; 372: 425-432 [published correction appears in Nature 1995; 374: 479].

4. Ahima RS, Flier JS. Leptin. Annu Rev Physiol 2000; 62: 413-437.

5. Karthikeyan BV, Pradeep AR. Gingival crevicular fluid and serum leptin: their relationship to periodontal health and disease. J Clin Periodontol 2007; 34: 467-472.

6. Purwar P, Khan MA, Mahdi AA, et al. Salivary and serum leptin concentrations in patients with chronic periodontitis. J Periodontol 2015; 86: 588-594.

7. Caton JG, Armitage G, Berglundh T, et al. A new classification scheme for periodontal and peri-implant diseases and conditions - introduction and key changes from the 1999 classification. J Clin Periodontol 2018; 45 Suppl 20: S1-S8.

8. Weir CB, Jan A. BMI classification percentile and cut off points. [Updated 2019 Dec 7]. In: StatPearls [Internet]. Treasure Island (FL): StatPearls Publishing; 2020 Jan. Available from: https:// www.ncbi.nlm.nih.gov/books/NBK541070/.

9. Silness J, Loe H. Periodontal disease in pregnancy. II. Correlation between oral hygiene and periodontal condition. Acta Odontol Scand 1964; 22: 121-135.

10. Loe H, Silness J. Periodontal disease in pregnancy. I. Prevalence and Severity. Acta Odontol Scand 1963; 21: 533-551.

11. Carranza FA, Newman MG, Takkie HH, Klokkevold PR. Clinica Periodontology. $7^{\text {th }}$ ed. Vol. 2. 2013, pp. 487-491.

12. Priya KY, Prathibha KM. Methods of collection of saliva - a review. Int J Oral Health Dent 2017; 3: 149-153.

13. Gangadhar V, Ramesh A, Thomas B. Correlation between leptin and the health of the gingiva: a predictor of medical risk. Indian J Dent Res 2011; 22: 537-541.

14. Paul RF, Hassan M, Nazar HS, Gillani S, Afzal N, Qayyum I. Effect of body mass index on serum leptin levels. J Ayub Med Coll Abbottabad 2011; 23: 40-43.

15. Shimada Y, Komatsu Y, Ikezawa-Suzuki I, Tai H, Sugita N, Yoshie H.The effect of periodontal treatment on serum leptin, interleukin-6, and C-reactive protein. J Periodontol 2010; 81: 1118-1123.

16. Kennedy GC. The role of depot fat in the hypothalamic control of food intake in the rat. Proc R Soc Lond B Biol Sci 1953; 140: 578-596.

17. Gundala R, Chava VK, Ramalingam K. Association of leptin in periodontitis and acute myocardial infarction. J Periodontol 2014; 85: 917-924.

18. Söderberg S, Ahrén B, Jansson JH, et al. Leptin is associated with increased risk of myocardial infarction. J Intern Med 1999; 246: 409-418.

19. Leyva F, Anker SD, Egerer K, Stevenson JC, Kox WJ, Coats AJ. Hyperleptinaemia in chronic heart failure. Relationships with insulin. Eur Heart J 1998; 19: 1547-1551.

20. Wallace AM, McMahon AD, Packard CJ, et al. Plasma leptin and the risk of cardiovascular disease in the west of Scotland coronary prevention study (WOSCOPS). Circulation 2001; 104: 3052-3056.

21. Beck J, Garcia R, Heiss G, Vokonas PS, Offenbacher S. Periodontal disease and cardiovascular disease. J Periodontol 1996; 67 (10 Suppl): 1123-1137.

22. Arnalich F, López J, Codoceo R, Jiménez M, Madero R, Montiel C Relationship of plasma leptin to plasma cytokines and human survivalin sepsis and septic shock. J Infect Dis 1999; 180: 908911.

23. Johnson RB, Serio FG. Leptin within healthy and diseased human gingiva. J Periodontol 2001; 72: 1254-1257.

24. Koh KK, Park SM, Quon MJ. Leptin and cardiovascular disease: response to therapeutic interventions. Circulation 2008; 117: 3238-3249.

25. Nakata M, Yada T, Soejima N, Maruyama I. Leptin promotes aggregation of human platelets via the long form of its receptor. Diabetes 1999; 48: 426-429.

26. Khorsand A, Bayani M, Yaghobee S, Torabi S, Kharrazifard MJ, Mohammadnejhad F. Evaluation of salivary leptin levels in healthy subjects and patients with advanced periodontitis. J Dent (Tehran) 2016; 13: 1-9.

27. Karthikeyan BV, Pradeep AR. Gingival crevicular fluid and serum leptin: their relationship to periodontal health and disease. J Clin Periodontol 2007; 34: 467-472.

28. Selvarajan S, Perumalsamy R, Emmadi P, Thiagarajan R, Namasivayam A. Association between gingival crevicular fluid leptin levels and periodontal status - a biochemical study on Indian patients. J Clin Diagn Res 2015; 9: ZC48-53.

29. Bełtowski J, Wójcicka G, Jamroz A. Leptin decreases plasma paraoxonase 1 (PON1) activity and induces oxidative stress: the possible novel mechanism for proatherogenic effect of chronic hyperleptinemia. Atherosclerosis 2003; 170: 21-29.

30. Parhami F, Tintut Y, Ballard A, Fogelman AM, Demer LL. Leptin enhances the calcification of vascular cells: artery wall as a target of leptin. Circ Res 2001; 88: 954-960.

31. Loffreda S, Yang SQ, Lin HZ, et al. Leptin regulates proinflammatory immune responses. FASEB J 1998; 12: 57-65.

32. Bozkurt FY, Yetkin Ay Z, Sütçü R, Delibaş N, Demirel R. Gingival crevicular fluid leptin levels in periodontitis patients with longterm and heavy smoking. J Periodontol 2006; 77: 634-640. 